\title{
¿Participa la esfera política en YouTube? Producción y consumo de información en la campaña electoral catalana de 2017
}

\author{
Does the political sphere participate in YouTube? Production and \\ consumption of information in the 2017 catalan electoral campaign
}

\author{
Dra. Marta Gil Ramírez, Universidad de Málaga (España) \\ martagr@uma.es | http://orcid.org/0000-0002-0577-5628 \\ Campus de Teatinos s/n, 29071, Málaga
}

\begin{abstract}
Resumen
La posibilidad de participación activa y directa en los contenidos que los nuevos canales digitales otorgan al ciudadano, obliga a replantear las estrategias de comunicación y marketing que, hasta ahora, venía utilizando la esfera política. Recientemente, numerosos estudios abordan el uso que partidos y candidatos hacen de las redes sociales. Twitter y Facebook son las plataformas sobre las que más se ha indagado. Otros canales de comunicación e interacción como YouTube, pese a competir en relevancia con las redes sociales anteriores, carecen de un recorrido académico que permita delimitar su importancia en la nueva configuración de la comunicación política digital. El objetivo de esta investigación es analizar el uso de YouTube como herramienta estratégica en período de campaña electoral, constatando el grado en que la esfera política participa en la producción de contenidos en esta plataforma y el tipo de consumo que se produce en la misma. Para ello, mediante análisis de contenido, se estudia la campaña electoral que precedió los comicios catalanes del 21D. Integran la muestra cuarenta vídeos, los diez con mayor número de reproducciones que atienden a las etiquetas de búsqueda: Junts per Catalunya, Ciutadans, Puigdemont y Arrimadas. Los resultados evidencian una producción muy baja por parte del ámbito de la política y una intervención media-baja en la creación de contenidos que, mayoritariamente, proceden de material generado por los medios de comunicación tradicionales, lo que apunta a un estadio embrionario en el aprovechamiento que los partidos y candidatos hacen de esta red social.
\end{abstract}




\begin{abstract}
The possibility of active and direct participation in the contents that the new digital channels give to the citizen, forces to rethink the communication and marketing strategies that, until now, the political sphere has been using. Recently, numerous studies address the use that parties and candidates make of social networks. Twitter and Facebook are the platforms on which more has been investigated. Other communication and interaction channels such as YouTube, despite competing in relevance with the previous social networks, lack an academic path that allows to delimit its importance in the new configuration of the digital political communication. The objective of this research is to analyze the use of YouTube as a strategic tool during the campaign period, stating the degree to which the political sphere participates in the production of content on this platform and the type of consumption that occurs in it. To do this, through content analysis, we study the electoral campaign that preceded the Catalan elections of 21D. The sample includes forty videos, the ten with the highest number of reproductions that serve the keywords: Junts per Catalunya, Ciutadans, Puigdemont and Arrimadas. The results show a very low production by the policy field and a medium-low intervention in the creation of content that, mostly, comes from material generated by traditional media, which points to an embryonic stage in the use that the parties and candidates make of this social network.
\end{abstract}

Palabras clave: comunicación política, YouTube, campaña electoral, redes sociales.

Keywords: political communication, YouTube, electoral campaign, social networks.

\title{
1. INTRODUCCIÓN
}

\subsection{Elecciones catalanas del 21D: interés político y seguimiento mediático}

El 21 de diciembre (en adelante 21D) de 2017 se celebraron en Cataluña elecciones autonómicas en un contexto singular (Coromina, Prado y Padilla, 2018), siendo estos comicios convocados por el Gobierno Central al amparo del artículo 155 de la Constitución Española como respuesta a la Declaración Unilateral de Independencia proclamada el 27 de octubre por Carles Puigdemont. A la candidatura a presidir el Parlament Catalán optaron 11 políticos, con la peculiaridad de que el candidato de Junts per Catalunya, Carles Puigdemont, se encontraba "huido" en Bruselas y el de Esquerra Republicana de Catalunya, Oriol Junqueras, estaba en prisión provisional acusado de sedición. Con tales circunstancias, la campaña electoral de los comicios del 21D se presenta como un acontecimiento particularmente interesante desde el punto de vista de la comunicación política.

El desencadenante -que no su origen- más próximo de este extraordinario contexto se podría situar, a efectos prácticos, en el 1 de octubre de 2017, fecha en la que se celebra, pese a la prohibición expresa del Gobierno Español y ante la declaración de ilegalidad del Tribunal Supremo, el Referéndum de autodeterminación catalán. Rodeado de gran polémica por las cargas policiales que se produjeron durante la jornada, este acontecimiento adquiere eco internacional tanto a través de los medios tradicionales, como en la globalidad de la web 2.0. 
Finalmente, Ciutadans es el partido que obtiene mayor número de votos (25,35 por ciento) y de escaños (36). Sin embargo, en estas elecciones no se trataba sólo de ser el partido vencedor. La presidencia de Cataluña se dirimía entre los partidos políticos del denominado bloque independentista (JxC, ERC y CUP) y los del opositor bloque constitucionalista (PP, PSOE y C'S). En el ecuador, el partido que se mantuvo entre los dos hemisferios: Catalunya en Comú-Podem.

Los comicios del 21D, así como todos los acontecimientos que los precedieron, tuvieron un extraordinario eco en los medios de comunicación y las redes sociales.

En España, a nivel mediático, las principales cadenas generalistas dedicaron un ingente número de horas a la cuestión catalana tanto en sus programas informativos, como en espacios de otra índole, alterando incluso sus programaciones en distintas ocasiones para ofrecer la última hora del conflicto.

Destaca, en este sentido, la programación de La Sexta que, con sus espacios "Al Rojo Vivo", "Más Vale Tarde", así como distintos especiales en prime time dedicados al desarrollo del Procés, cosechó cifras históricas de audiencia. No en vano, La Sexta fue la única televisión generalista que, junto a la cadena pública RTVE, organizó un debate durante la contienda electoral. A nivel regional, TV3 también emitió un debate que contó con una destacada cuota de pantalla ${ }^{1}$. La crisis catalana provocó un espectacular crecimiento en la audiencia de la televisión pública autonómica².

En relación a los socialmedia, el estudio publicado por el periódico El País (20 de diciembre de 2017) o el que se puede consultar a través del portal Acceso (19 de diciembre de 2017), dan cuenta de la importante repercusión que esta campaña electoral tuvo en redes sociales como Twitter o Facebook. Los datos recogidos en el estudio "21D: la campaña electoral en redes sociales" (Acceso, 2017) indican que en Twitter se produjeron más de 3 millones de conversaciones, aumentando el volumen habitual en un 236 por ciento durante el periodo previo a los comicios.

A nivel académico, los trabajos de Pérez-Curiel y García-Gordillo (2018) y Coromina et al. (2018) ponen el foco en estas elecciones. El primero aborda las estrategias y efectos de la comunicación política desarrollada en Twitter y el segundo profundiza sobre las emociones a las que apelan los partidos políticos a través de las narrativas desplegadas en Facebook durante la campaña electoral.

En la última década, la producción científica en torno a la utilización por parte de la esfera política de las redes sociales, especialmente en periodos electorales, ha sido prolífica. Sin embargo, la mayor parte de la investigación ha tomado como contexto las redes sociales Twitter y Facebook, desatendiendo otras plataformas cuyo análisis puede generar una perspectiva más global y completa de la importancia que han adquirido los nuevos canales digitales en la comunicación política 2.0.

Este estudio se propone examinar, a través del análisis de contenido de los vídeos más reproducidos en YouTube sobre Junts per Catalunya y Ciutadans, así como sus respectivos 
candidatos, el uso de este canal como herramienta estratégica en período de campaña electoral, constatando el grado en que la esfera política participa en la producción de contenidos en esta plataforma y el tipo de consumo que se produce en la misma. Las evidencias obtenidas permitirán sumar al incipiente abordaje científico del rol que juega el ámbito político en los contenidos en YouTube en comparación con otras redes sociales donde este aspecto ha sido ya más indagado, aportando a su vez resultados empíricos que, junto a los de futuras investigaciones, contribuyan a delimitar la relevancia de esta plataforma en la configuración de la nueva política digital.

\subsection{La eclosión del prosumidor en Comunicación Política}

Años atrás, la política se ha servido, en los períodos de campaña electoral, de mítines, reparto de octavillas y debates entre candidatos como principales herramientas para dar a conocer sus programas electorales a la ciudadanía. Todos estos actos han contado tradicionalmente con la cobertura y difusión por parte de los medios de comunicación de masas que, sujetos a una mayor o menor tendencia ideológica, trasladaban la información de campaña a la sociedad civil. De esta forma, "gobiernos y corporaciones han acaparado los canales de comunicación para afianzar su poder" (Castells, 2012, p. 29). Hasta la llegada de Internet y la posterior eclosión de las redes sociales, la esfera pública contaba con escasa participación en este ámbito. Los socialmedia transforman por completo estas relaciones de poder dando al ciudadano la posibilidad de incidir de manera directa en los otros dos polos que configuran la triangulación y ampliando en número y tipología los actores que interactúan y negocian en el campo de la comunicación política (Casero, 2018). "La actual consolidación de una nueva galaxia mediática está provocando cambios fundamentales en múltiples ámbitos como la política, la economía y la comunicación, que ven alteradas sus estructuras" (Feenstra y Casero, 2012, p. 129). "Gracias a Internet, los ciudadanos, operando más allá de los medios convencionales, han llegado a afectar y condicionar, en un contexto político agitado, el significado y el flujo de la información de actualidad" (Feenstra y Casero, 2012, p. 137). Para Loosen (como se citó en Gil, 2015, p. 24), "actualmente estaría surgiendo una tercera concepción, según la cual la audiencia sería un 'conjunto de redes empoderadas', compuesta por sujetos activos que colaboran en la producción y diseminación de información, con la ayuda de redes de medios digitales".

Estaríamos hablando de lo que hoy en día se conoce como prosumidores o prosumers (Toffler, 1980). En este escenario, partidos políticos, medios de comunicación y sociedad civil se posicionan al mismo nivel al tener, todos ellos, la posibilidad de producir y consumir contenido político en la web 2.0. Así, en el ámbito de la comunicación política, los tres actores participantes tienen ahora la misma posición de partida. Los hechos se pueden contrastar, debatir y refutar sin que los poderes fácticos de antaño dispongan de mucho más que de otro altavoz digital de igual calado que el que puede tener cualquier otra persona de la Red (Polo y Cárdenas, 2014).

Esto es, el prosumidor en comunicación política, podría actualmente ocupar, bien la esfera política, la mediática o la pública, ya que los tres actores se han lanzado a producir contenidos en la web 2.0 además de consumirlos. Ahora bien, la literatura académica reciente asocia el término prosumidor a la esfera pública (véanse a modo de ejemplo: Aparici y García-Marín; 2018; 
Gil, 2015; Jordán, Arias y Samaniego, 2017), una vinculación tan extendida que se ha validado. Entendemos que valdría la pena que futuros estudios se replantearan la adjudicación de este término (prosumer) a una acepción limitada a la esfera pública, a la producida por la sociedad civil, ya que actualmente así se entiende: desde la perspectiva novedosa del rol activo adquirido por los ciudadanos, previamente receptores pasivos.

La posibilidad de participación e intervención ciudadana en el contenido político ha hecho tambalear la tradicional estructura de poderes, obligando a los partidos políticos a descender a las nuevas arenas de batalla -las redes sociales-, máxime en los períodos de campaña electoral, para tratar de arañar votos. Estos cambios están rompiendo el monopolio de las élites periodísticas y políticas sobre la construcción de las noticias (Casero-Ripollés, 2010). Ambas esferas se ven obligadas a interaccionar con los ciudadanos y los nuevos actores informativos en la producción y distribución de la información (Deuze, 2007; Jenkins, 2006). El ambiente informativo, antes centrado en las relaciones entre un número reducido de sujetos (periodistas, políticos y spin doctors), ahora engloba a múltiples grupos e individuos (Sampedro y Resina, 2010). "La comunicación entre los sujetos ya no está mediada sino que es directa (...) En algunas ocasiones se considera que este sistema está configurando un nuevo espacio público" (Espino, 2014, p. 42).

Esta situación modifica tanto las estrategias de comunicación de los propios partidos políticos, como los comportamientos de los usuarios (Berrocal, Martín y Gil, 2017). La emergencia de este nuevo ambiente informativo supone un cambio de paradigma. Desde un panorama caracterizado por el control de la información, ejercido de arriba-abajo por parte de las élites periodísticas y políticas para mantener el orden social, se está pasando a una dinámica dominada por el caos (McNair, 2006). "En este nuevo escenario la abundancia de información predomina frente a la escasez, la difusión de noticias de muchos-a-muchos (many-to many) frente a la transmisión de uno-a-muchos (one-tomany)" (Feenstra y Casero, 2012, p. 131), primando la descentralización de la información (Casero, 2018).

Ocurre que este nuevo contexto social es un terreno desconocido para la vieja política, acostumbrada a la arenga y la oratoria, mediada en la mayoría de las ocasiones, por la pantalla de plasma. Los socialmedia, y el caso concreto de YouTube es buen exponente de ello, manejan sus propias reglas. En la Red se impone un lenguaje directo, corto, con elementos audiovisuales o gráficos llamativos, es decir, unos modos de expresión política equidistantes al tradicional hacer de la política mediatizada. En las redes sociales la sociedad civil es la protagonista, o al menos, ese fue su sentido original, un espacio de debate público donde todo ciudadano podía expresarse libremente al modo del ágora griega. "Los actores sociales y ciudadanos de todo el mundo se están articulando a través de las nuevas capacidades de las redes de comunicación con la finalidad de hacer avanzar sus proyectos, defender sus intereses y reafirmar sus valores" (Castells, como se citó en Espino, 2014, p. 42).

La figura de los 'prosumidores' como sujetos 'empoderados' con actitud 'emprendedora' cuyas creaciones no solo generan riqueza económica, sino que transforman el entorno 
político y social, se convierte en una pieza clave en la articulación social que surge del impacto de las nuevas tecnologías. (Gil, 2015, p. 30-31)

Ahora bien, ¿están la política y sus actores protagonistas dispuestos a afrontar el contacto directo que se genera en estos nuevos canales de comunicación? ¿Sabrá la esfera política dar el salto de dirigirse a una masa que no responde más que con el voto, a hacerlo respecto a una infinita red de individuos que interpelan a tiempo real el contenido que se propone? A este respecto Abejón, Sastre y Linares (2012) se cuestionan: ¿están los políticos preparados para lidiar con los individuos y no con las masas? Polo y Cárdenas (2014) apuntan a la potencialidad de las redes sociales como herramienta para la concurrencia pública de ideas entre la esfera política y la pública. Espino (2014) muestra como distintos intelectuales consideran que, con los procesos de diálogo entre pares que se desarrollan a través de las redes sociales, se puede volver a configurar la democracia deliberativa perdida en el siglo XX a favor de las democracias representativas que dependen de los medios de comunicación masiva.

\subsection{El estudio de la participación de la esfera política en redes sociales}

Hace más de un lustro, De Moragas (2011) ya apuntaba a la investigación centrada en las condiciones de participación que se abren con la aparición de los nuevos medios, como una probable evolución en la comunicación política. Desde las elecciones presidenciales de 2008 en Estados Unidos, grandes universidades y centros de investigación se han centrado en cómo influyen o se relacionan las redes sociales y los votantes (Deltell, 2012).

La utilización por parte de la esfera política de los nuevos canales digitales, especialmente en periodos electorales aunque no exclusivamente, ha despertado gran interés entre la comunidad científica en la última década. La mayoría de los estudios recientes que abordan a nivel empírico esta cuestión constatan la falta de aprovechamiento por parte de los líderes y partidos políticos de estos canales de comunicación.

Twitter es la red social sobre la que ponen el foco la mayor parte de los trabajos previos (Alonso y Casero, 2018: Alonso, Marcos y Casero, 2016; Catalina, López-de-Ayala y Fernández-Fernández, 2015; López-Abellán, 2012; López-Meri, 2016; Miquel, Alonso y Marcos, 2017; Pérez-Curiel y García-Gordillo, 2018; Polo y Cárdenas, 2014; Quevedo, Portalés y Berrocal, 2016). Casero (2018) achaca esta circunstancia a la facilidad de acceso a los datos en esta red de microblogging, lo que facilita la investigación; su marcado carácter informativo más cercano al ámbito político que en otras plataformas o la alta presencia en ella de políticos y periodistas, lo que le confiere un status de centralidad en la información política. Es en Twitter, donde únicamente encontramos algunas investigaciones que apuntan hacia una leve evolución en positivo en relación a la participación de la esfera política y su interacción con los contenidos producidos por la ciudadanía (Criado, Martínez y Silván, 2013; Graham, Broersma, Hazelhoff y Van’t-Haar, 2013; Jivkova, Requeijo y Padilla, 2017). 
La participación y aprovechamiento que los partidos y líderes políticos hacen de Facebook, aunque en una proporción menor que en el caso de Twitter, también ha sido objeto de estudio en numerosos trabajos recientes (Abejón y Mayoral, 2017; Ballesteros y Diez, 2018; Fenoll y Cano, 2017; Muñiz, Dader, Téllez y Salazar, 2016; Rodríguez-Fidalgo, Ruiz, Paíno y Jiménez, 2017; Tuñez y Sixto, 2011). Otras investigaciones abordan Facebook y Twitter conjuntamente (Abejón et al., 2012; Espino, 2014) y encontramos también ejemplos en los que el análisis se realiza sobre la relación gobernantes-ciudadanía en websites o blog, de forma aislada o junto a otras redes sociales (Almansa y Castillo, 2014; Aparaschivei, 2010; Dader, 2009; Deltell, 2012; Mascheroni y Matoni, 2012; Schweitze, 2012). En todos estos casos, la literatura académica apunta hacia un uso unidireccional de estas plataformas digitales, empleadas por partidos y líderes a modo propagandístico en lugar de para el intercambio de opiniones, desaprovechando así las oportunidades de comunicación y marketing que brindan estos nuevos canales.

YouTube sin embargo, pese a ser uno de los principales espacios digitales de intercambio de contenido y mayor interacción, situándose actualmente como el sitio web con mayor número de visitas $^{3}$, es, sin duda, la red social cuyo uso como herramienta estratégica en comunicación política ha sido menos indagado. Tanto en el contexto internacional como en el español, los trabajos empíricos que abordan la participación del poder político en este canal online son aun escasos. Los trabajos académicos recientes fuera de nuestras fronteras (Borah, Fowler, y Ridout, 2018; Church, 2010; Sohal, y Kaur, 2018; Vesnic y Van Bauwel, 2014 ) se han centrado principalmente en el análisis de la publicidad política en esta red social en distintos contextos electorales, ahondando en la naturaleza y tipología de este tipo de contenidos. Este objeto de estudio, aunque en sintonía con el ámbito que abarca la comunicación política en YouTube, se aleja del examen de aspectos concretos que persigue la presente propuesta. En el ámbito nacional, las investigaciones de Berrocal et al. (2012a; 2012b; 2014; 2016; 2017) constituyen un nítido referente entre los estudios que examinan algunos de los aspectos de la comunicación política que se consume y difunde a través de YouTube. Centrados en el análisis del infoentretenimiento, estos trabajos inciden igualmente en la escasa intervención e implicación del ámbito político en esta red social. Otro trabajo que permite profundizar en el uso que la esfera política hace de YouTube es el de VázquezSande (2016). El autor aborda el contexto de las elecciones municipales gallegas de 2011, evidenciando una tendencia, todavía embrionaria en la plataforma de vídeos online, al recurso de contenidos personales en forma de relato. Por último cabe destacar la reciente aportación de Rodríguez-Breijo, Gallardo y Sierra (2018) en la que se contextualizan distintos aspectos de la información política que circula en YouTube y como novedad, se compara el peso de este tipo de contenidos con el resto de temáticas que se pueden encontrar en la plataforma de vídeos online.

Atendiendo al hecho de que cada red social posee unas características diferentes que provocan dinámicas y efectos distintos (Casero, 2018), parece evidente la carestía de estudios que contribuyan a reforzar el aún embrionario abordaje de la comunicación política en YouTube, como es la pretensión de esta investigación. A esta necesidad se suma el hecho de que, en el ámbito de la academia española, sólo tres de los estudios que toman como contexto la plataforma de vídeos online (Berrocal et al., 2016; 2017; Vázquez-Sande, 2016) examinan algún aspecto de la comunicación política en periodo electoral, arrojando conclusiones dispares: unas veces 
coincidentes con las del presente trabajo, otras veces no. Los resultados obtenidos sobre el uso que la esfera política hace de este canal en período de campaña electoral y el rol que juega en la producción de contenidos son escasos y, de momento, no son concluyentes. Esta investigación trata de contribuir a llenar este vacío.

\section{OBJETIVOS GENERALES Y ESPECÍFICOS}

El objetivo general de esta investigación es analizar el uso de YouTube como herramienta estratégica en períodos de campaña electoral constatando el grado en que la esfera política participa en la producción de contenidos en esta plataforma y el tipo de consumo que se produce en la misma.

Atendiendo a este objetivo general y a la revisión teórica reflejada anteriormente, nos proponemos los siguientes objetivos específicos:

01. Analizar el nivel de participación de los partidos políticos como productores de contenido en YouTube. En este sentido, se plantea la comparación de la actividad de la esfera política frente a la mediática y a la pública para detectar quién ostenta la autoría del discurso político en YouTube durante el período de campaña analizado.

02. Comprobar si el contenido publicado en YouTube ha sido adaptado al lenguaje propio de las redes sociales ${ }^{4}$, es decir, analizar si la intervención en la creación de contenidos difundidos en la plataforma de vídeos online por parte de los distintos actores que participan de la comunicación política (medios, ciudadanía y política) es alta, media o baja, examinando la procedencia del material audiovisual y el nivel de manipulación del mismo, con especial atención al publicado por partidos políticos. Este objetivo trata de contrastar resultados obtenidos por trabajos previos que apuntan hacia una incipiente "hibridación" entre los nuevos y viejos medios (Alonso et al., 2016; Chadwick, 2017; López-Meri, Marcos y Casero, 2017) o hacia una "transmediación" (Cheong y Lundry, 2012) desde contenidos televisivos hacia esta red social, transformando el contenido con una pequeña acción textual, visual y/o sonora (Berrocal, 2014).

03. Examinar la temática y categoría ${ }^{5}$ de los vídeos publicados en YouTube, prestando especial atención a los difundidos por partidos políticos. Esta premisa, de nuevo trata de corroborar resultados precedentes que apuntan hacia un descenso considerable del politaiment a favor de contenidos institucionales durante periodos electorales (Berrocal et al., 2016; 2017) frente a la primacía del infoentretenimiento en campaña permanente (Berrocal et al., 2012a; 2012b; 2012c; 2014).

04. Constatar si el fenómeno de la personalización, evidenciado en diversos trabajos sobre comunicación política en televisión (véanse a modo de ejemplo: Berrocal, 2003; Dader, 1990), se traslada al ámbito de YouTube y tiene reflejo en los vídeos examinados. Respecto a este item, la literatura previa ofrece conclusiones ambiguas apuntando, una veces hacia 
la ausencia de este fenómeno (Berrocal et al., 2016), otras a una clara tendencia hacia él (Berrocal et al., 2017).

A través de estos objetivos específicos se persigue profundizar en el tipo de prosumición (producción y consumo) que se produce en YouTube durante un periodo de campaña electoral por parte de los tres actores que intervienen en la comunicación política (esfera mediática, pública y política) para determinar, en base a la comparación de la actividad de todos ellos, el nivel y la tipología de la intervención de los partidos y líderes políticos en los contenidos difundidos en esta red social.

\section{METODOLOGÍA}

La investigación realizada es un análisis de contenido cuantitativo y descriptivo que aborda la participación de la esfera política en la producción de contenido electoral en la red social YouTube mediante el análisis de 40 vídeos que se corresponden con los 10 que recogen mayor número de reproducciones bajo las etiquetas: Junts per Catalunya, Ciutadans, Puigdemont y Arrimadas.

La elección de estas etiquetas de búsqueda viene determinada por tratarse de las dos formaciones y sus respectivos candidatos, que obtuvieron mayor número de votos $(25,35$ por ciento Ciutadans y 21,66 por ciento Junts per Catalunya) y de escaños (36-34), aglutinando entre ambos la representación de más de la mitad del Parlament. Otra motivación de esta selección reside en que se trata de los partidos que abanderan los dos bloques que pugnaron en las elecciones del 21D por el gobierno catalán: el independentista y el constitucionalista. Esta confrontación representó el núcleo del debate electoral por delante de cualquier otro tema de interés público (Pérez-Curiel y García-Gordillo, 2018).

Por otra parte, la elección de YouTube y no otro tipo de red social, viene determinada por ser esta plataforma la que cuenta con el abordaje científico más inicial en cuanto al objeto que nos ocupa. Este hecho está quizás relacionado con que, en otras redes sociales, distintas aplicaciones (véanse por ejemplo: Trendsmap, Blitzmetric, Twtrland, Wisemetrics, TweetReach) facilitan la labor del investigador ofreciendo métricas de un amplio abanico de datos a nivel cuantitativo. No ocurre así en YouTube. Como constata Deltell (2012, p. 9) en su trabajo sobre las estrategias de comunicación política implementadas por Equo en Facebook, Twitter y YouTube durante la campaña previa a las elecciones de 2011 en España, la plataforma de vídeos "es de todas las herramientas analizadas la más compleja para evaluar".

En esta investigación la base de datos se confeccionó de forma manual, estableciendo listados con los enlaces, disponibles en cualquier momento, a los contenidos de los vídeos analizados en YouTube, así como tablas (fichas) que permitieran la codificación y registro de los datos a examinar.

La muestra se recoge el 20 de diciembre de 2017 a las 23.00 horas. Se establecen los siguiente parámetros de búsqueda bajo las etiquetas supra mencionadas: "fecha de subida: este mes", 
"tipo: vídeo", y “orden: número de visualizaciones". Se obtienen los siguientes resultados filtrados: para Junts per Catalunya, 2.090 entradas; 1.230 para Ciutadans, para Puigdemont, 7.020 y 4.470 para Arrimadas. Con el fin de acotar la muestra se toma como nuevo criterio de filtro, atender, en cada caso, a los 10 vídeos más veces reproducidos. Los contenidos examinados suponen la visualización y el análisis de $\mathbf{4 7 0}$ minutos de audiovisual. En el transcurso de la investigación y de la revisión por parte de los diferentes codificadores se descarta un contenido por el siguiente motivo: este vídeo no está disponible ${ }^{6}$. Así, finalmente la muestra queda delimitada a 39 vídeos.

El diseño de la ficha de análisis incluye distintas variables y categorías cuya inclusión se apoya en la pertinencia al objeto de estudio constatada en estudios previos de gran difusión académica (Berrocal et al., 2012a; 2012b; 2014; 2016; 2017), adaptándolas a las peculiaridades y propósitos de esta investigación concreta. Así, la confección de la plantilla de análisis responden a las siguientes preguntas de investigación:

- ¿Quién es el autor de los vídeos analizados? Que se concreta en las siguientes preguntas: ¿Quién publica el vídeo en YouTube: usuario común, medio de comunicación, partido político? Los medios de comunicación que publican ¿son españoles o extranjeros? Quien publica el vídeo ¿es quien lo ha creado? ¿El prosumidor produce el vídeo específicamente para YouTube o lo traslada directamente desde material producido por medios de comunicación? ¿Se realizan modificaciones -imagen, sonido, rotulaciones, etc.- sobre el material original para adaptarlo al lenguaje propio de las redes sociales?

- ¿Cómo son los vídeos analizados? Con las siguientes cuestiones: ¿Cuántas visualizaciones acumulan? ¿Es un vídeo político, publicitario, de humor o de crítica satírica? ¿Contienen componente de "infoentretenimiento"?

- Finalmente, ¿qué reflejan los vídeos analizados? Con los interrogantes que siguen: ¿Cuál es la temática del vídeo? ¿Quién es el protagonista? ¿Predominan los aspectos personales y anecdóticos de los actores políticos sobre la información electoral?

La plantilla de análisis ha sido confeccionada a partir de variables independientes y dependientes, con posibilidad de respuesta múltiple o dicotómica. La ficha fue aplicada por dos codificadores con un índice de concordancia sustancia $(K=0,73)$.

\section{RESULTADOS}

\subsection{La participación de la esfera política como productor de contenido en YouTube}

Conocer en profundidad la autoría en la producción de los vídeos analizados es uno de los objetivos de esta investigación. Así distinguiremos entre quien crea el contenido y quien lo publica. En el intento de esclarecer la tipología de la producción en YouTube, también es preciso atender a la diferenciación entre aquellos contenidos que han sido creados específicamente para este 
tipo de plataforma o adaptados a ella y aquellos que son subidos a la Red tomando como base, material desde los medios de comunicación tradicionales sin modificación alguna.

Los vídeos analizados se corresponden con 22 titulares de cuentas de YouTube, dado que muchos de ellos "suben" a la red más de un vídeo. De estos, el $50 \%$ son usuarios comunes, el $36 \%$ medios de comunicación y el $14 \%$ partidos políticos. En los usuarios comunes diferenciamos entre los que denominamos "particulares": aquellos que ofrecen algún dato que los identifica como dueños del canal a título propio; y los que mencionamos como "anónimos": usuarios que no ofrecen información sobre sí mismos o sobre su cuenta de YouTube. Así, entre los usuarios comunes el $55 \%$ se corresponde con particulares y el $45 \%$ con anónimos. En el caso en el que los contenidos son producidos por medios de comunicación, distinguimos entre aquellos que emiten sólo en lengua catalana, los que publican exclusivamente en español y aquellos que lo hacen tanto en catalán como en castellano. En este sentido, un $38 \%$ de los medios de comunicación que "cuelgan" vídeos relacionados con la campaña examinada, utilizan la lengua catalana únicamente, un $37 \%$ emplean sólo el castellano y un $25 \%$ ofrecen ambas versiones. En relación con la producción de contenido por parte de la esfera mediática destacan, por su ausencia, los medios de comunicación extranjeros, dadas las especiales condiciones en que se celebra la campaña electoral analizada y la implicación europea en la cuestión catalana.

\section{Gráfico 1. Distribución de los vídeos en función de quién los publica Fuente: elaboración propia}

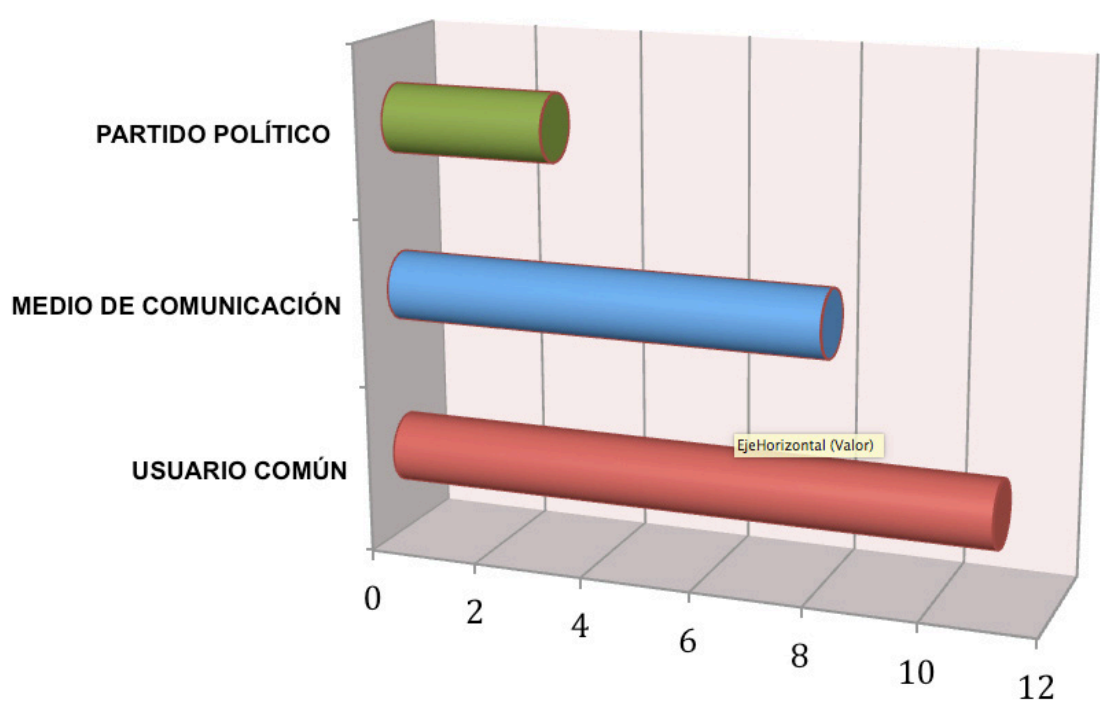

Respecto a los partidos políticos que producen contenido en YouTube durante la campaña electoral objeto de estudio, encontramos representadas a las dos formaciones analizadas bajo etiquetas con su nombre, esto es Junts per Catalunya y Ciutadans. Un tercer partido político que 
participa de la comunicación difundida a través de la plataforma social es Partit Demòcrata (partido demócrata europeo catalán). Ahora bien, estas organizaciones políticas tan sólo publican el 13\% de los vídeos analizados. Los medios de comunicación "suben" el $31 \%$ de los contenidos y los usuarios comunes son los que divulgan mayor número de informaciones (56\%), copando más de la mitad de los vídeos considerados.

Los medios de comunicación están presentes en 3 de las etiquetas propuestas: Junts per Catalunya, Puigdemont y Ciutadans. Los partidos políticos, por su parte, actúan como productores, sólo bajo las etiquetas que los definen, esto es, Junts per Catalunya y Ciutadans. Los usuarios comunes, que ostentan, como hemos visto, la hegemonía del discurso al menos en relación con el número de vídeos publicados, participan de las cuatro etiquetas propuestas.

En relación a la cuestión sobre si el contenido difundido a través de YouTube respecto a la campaña electoral del 21D es generado por el mismo usuario que lo publica o por un productor distinto, en un $46 \%$ de los vídeos analizados coincide el creador del contenido con el usuario que lo publica. En el $54 \%$ restante, el origen del material proviene de una fuente distinta al titular que lo difunde. Atendiendo a este último supuesto, el material del contenido proviene, en el $38 \%$ de los casos, de medios de comunicación, en mismo porcentaje (38\%) de imágenes fijas de Internet, un $19 \%$ de los vídeos toman como base, material proporcionado por partidos políticos y en una sola ocasión el audiovisual proviene de una grabación realizada con un teléfono móvil a nivel particular.

\section{Gráfico 2. Distribución del origen del material de los vídeos Fuente: elaboración propia}

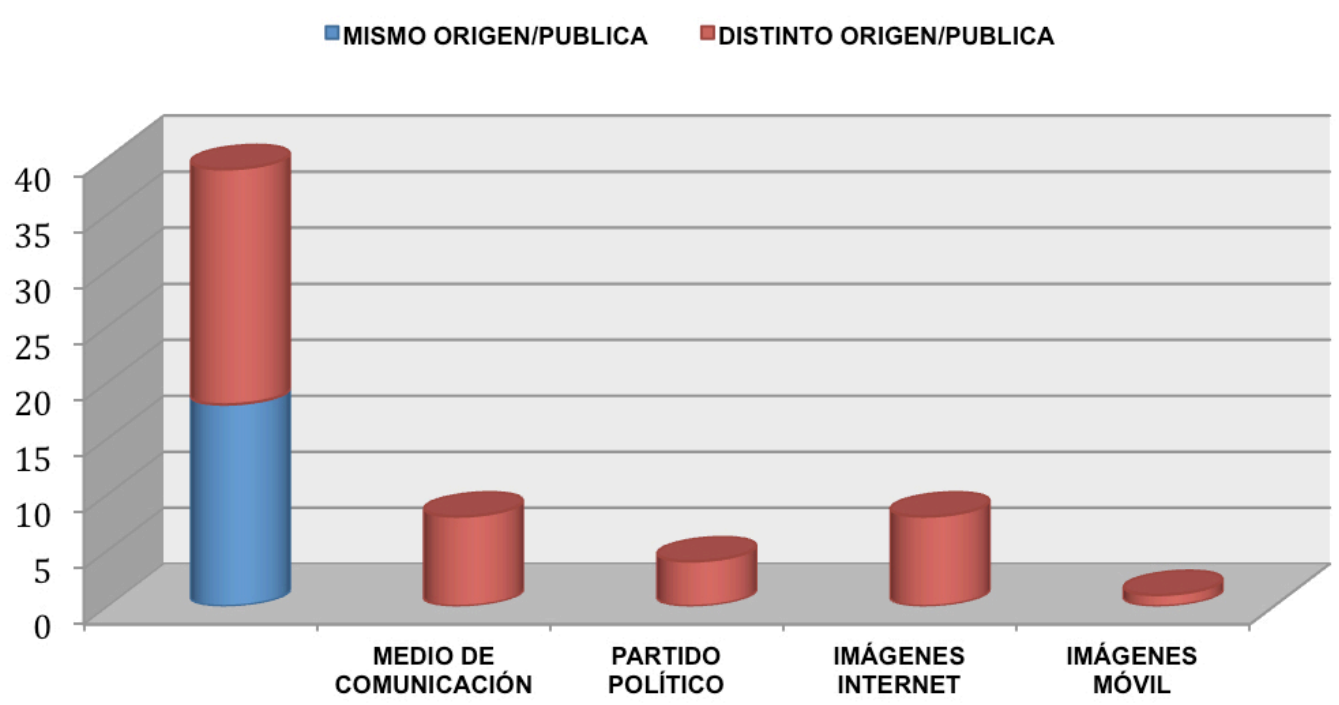

Por otro lado, existe bastante equilibrio entre los vídeos que se adaptan al lenguaje propio de YouTube (49\%) y aquellos que replican, sin modificación, el contenido generado por medios de 
comunicación tradicionales (51\%). Los vídeos que muestran modos expresivos propios de las redes sociales ${ }^{7}$ se corresponden -salvo una excepción- con el contenido publicado por los usuarios comunes. En este sentido, entre los contenidos que muestran alguna de estas características, tan sólo 1 se puede considerar de autoría propia del usuario que lo publica, mientras que el 95\% tienen su origen en medios de comunicación y han sido posteriormente manipulados, retocados para adaptarse a los códigos de los socialmedia. Los medios de comunicación y partidos políticos no adecuan el contenido a las características expresivas de YouTube, sino que lo trasladan a esta plataforma con un formato más propio de los medios de comunicación de masas, que de los nuevos canales de comunicación 2.0.

\subsubsection{Tipología del contenido producido en YouTube}

El vídeo, de entre los analizados, que se sitúa en el primer puesto en número de visualizaciones y que presenta, en el momento del estudio, 450.389 , se trata del spot de campaña de Ciutadans "Ara sí, Votarem". Por su parte, el que cuenta con menos reproducciones, 188 en el momento del análisis, refleja unas declaraciones de Arrimadas afirmando que "Ciutadans es el único partido que puede ganar el voto al independentismo" . En medio de estos dos extremos se hayan los 39 vídeos analizados. De éstos, una abrumadora mayoría, el 59\%, tienen un componente político predominante. Ejemplo de ello son las entrevistas realizadas a los candidatos, Puigdemont y Arrimadas, y en las que éstos tienen la oportunidad de comentar la actualidad política catalana ${ }^{10}$. A esta categoría le sigue la que hemos denominado "publicidad electoral", en la que se enmarcan el $23 \%$ de los vídeos considerados ${ }^{11}$. Un $10 \%$ tienen un claro componente humorístico ${ }^{12}$, mientras que el $8 \%$ del contenido es claramente una crítica satírica sobre el conflicto catalán o sobre la actuación de los actores protagonistas en el mismo ${ }^{13}$. En esta investigación se entienden estas cuatro categorías como se describe en la tabla 1.

Tabla 1. Descripción de categorías según el modo predominante en el vídeo

\begin{tabular}{|l|l|}
\hline $\begin{array}{l}\text { Información } \\
\text { Política }\end{array}$ & $\begin{array}{l}\text { Categoría que agrupa los vídeos que dan cuenta de un hecho, noticia o } \\
\text { circunstancia políticos de manera objetiva. }\end{array}$ \\
\hline $\begin{array}{l}\text { Publicidad } \\
\text { Electoral }\end{array}$ & $\begin{array}{l}\text { Bajo esta categoría se aglutinan aquellos vídeos en los que el material es } \\
\text { generado, difundido o proporcionado por un partido político. Véanse spots de } \\
\text { campaña, material sobre mítines, actos de partido, etc. }\end{array}$ \\
\hline Humor & $\begin{array}{l}\text { Categoría relacionada con el humorismo, resaltando el lado risueño, alegre y } \\
\text { lúdico del vídeo. }\end{array}$ \\
\hline Crítica Satírica & $\begin{array}{l}\text { En esta categoría se agrupan los vídeos cuya carga principal es la sátira, es } \\
\text { decir, la expresión de un juicio de valor en el que se muestra indignación hacia } \\
\text { alguien o algo con un propósito moral, de entretenimiento o de burla. }\end{array}$ \\
\hline
\end{tabular}

Fuente: elaboración propia a partir de Berrocal et al. (2012b) 
En cuanto a que los vídeos, independientemente de que correspondan a una u otra categoría, se puedan considerar de "infoentretenimiento", el resultado del análisis arroja que sólo en el 10\% de los contenidos que integran la categoría "humor" se observa esta condición.

\subsection{El consumo del contenido producido en YouTube}

Analizamos a continuación cuáles son las temáticas de los vídeos más reproducidos en YouTube bajo las etiquetas propuestas en esta investigación y cuáles son los actores protagonistas de estos contenidos. Tratamos con ello de establecer una visión general de cuál ha sido el interés que ha motivado el consumo de vídeos en YouTube en relación con el tema objeto de este estudio: la campaña electoral previa a las elecciones del 21D en Cataluña.

En cuanto a los temas que recogen los vídeos analizados, al margen de las temáticas propias de una contienda electoral como los spots de campaña o la información sobre mítines y actos electorales, presentes en el $23 \%$ de la muestra, tan sólo un vídeo versa sobre aspectos de la campaña ajenos a la promoción de uno u otro partido/candidato en particular, recogiendo información sobre las encuestas publicadas sobre intención de voto. El resto de temáticas se alejan de lo que podríamos denominar información de interés electoral. Destaca un bloque, que supone el $18 \%$ de la muestra, publicado principalmente por usuarios comunes (ciudadanos particulares), en el que se narran aspectos relacionados con la financiación de la estancia de Puigdemont en Bélgica o con el finiquito que éste recibe tras su cese como President. Algunos de los contenidos analizados versan sobre aspectos personales o anecdóticos de los candidatos, como el estilo, los gestos, la ropa de Puigdemont o la carpeta que Arrimadas forró en su juventud con la imagen de Pep Guardiola. Cabe mencionar también un contenido que se repite y que obtuvo gran repercusión en otras redes sociales, como es el vídeo -grabado con un móvil particular- en el que un supuesto militar amenaza a Puigdemont e Iglesias desde un tanque. Otros asuntos que se muestran en los vídeos analizados, aunque con escasa repercusión en términos porcentuales, son las declaraciones de Turrull tras salir de prisión, la crítica del periodista Carlos Herrera a Puigdemont, o las declaraciones en las que Gispert "envía a Cádiz a Arrimadas".

En cuanto a quiénes protagonizan los vídeos más visualizados en YouTube bajo los parámetros propuestos y distinguiendo entre candidatos y partidos, el análisis evidencia una clara tendencia a la personalización política, dado que Puigdemont y Arrimadas no sólo son protagonistas en los vídeos analizados bajo las etiquetas propuestas con estos apellidos, sino que copan parte de los contenidos más reproducidos bajo las etiquetas definidas por sus partidos políticos (Junts per Catalunya y Ciutadans). Puigdemont es protagonista en el $36 \%$ de los vídeos de la muestra, mientras que Arrimadas lo es en $28 \%$. Los partidos políticos, entendidos éstos como sujetos de la acción, obtienen un discreto segundo lugar. Junts per Catalunya es central tan sólo en una ocasión (3\%) frente al 15\% de los vídeos en los que Ciutadans figura en primer término. 


\section{DISCUSIÓN Y CONCLUSIONES}

Atendiendo a los resultados de esta investigación, y tratando de responder a los objetivos planteados, proponemos las siguientes conclusiones:

Se corrobora en YouTube la tendencia evidenciada en trabajos precedentes en relación a otras redes sociales como Twitter y Facebook ${ }^{14}$ sobre el escaso aprovechamiento que la esfera política hace de estos espacios digitales como herramienta estratégica en períodos de campaña electoral. Esta investigación se suma, por tanto, a otras "llevadas a cabo en distintas partes del mundo, que desmienten que las campañas políticas se hayan transformado gracias a las nuevas herramientas de la internet social" (Espino, 2014, p. 40). Los datos obtenidos indican que los políticos no participan en el nuevo modelo de convergencia a través de esta plataforma de vídeos online. El caso analizado demuestra que los responsables de la comunicación política electoral utilizan YouTube meramente como altavoz y repetidor de mensajes y consignas generados con los modos propios de los medios tradicionales, sin adaptarlos para una audiencia online.

Los ciudadanos particulares son mayoritarios en la producción de contenido sobre comunicación política en la campaña previa a las elecciones catalanas del 21D, lo que podría entenderse como una vuelta hacia la democracia deliberativa habermasiana perdida en favor de la democracia representativa protagonizada por los medios de comunicación de masas. Este dato apunta al empuje de la sociedad civil, en su rol de prosumidora, como elemento transformador de las relaciones de poder clásicas que han dominado la tradicional comunicación política.

Esta investigación refuerza algunas de las conclusiones de trabajos precedentes (Berrocal et al., 2012a; 2012b; 2012c; 2014), que apuntan a una adaptación media-baja en la producción de contenidos al lenguaje propio de la plataforma YouTube, caracterizado por mensajes directos, cortos y protagonizados por elementos audiovisuales o gráficos llamativos de creación propia. Se tiende, de forma predominante, a trasladar a esta plataforma, material generado por medios de comunicación de masas con pequeñas alteraciones, manipulaciones -textuales o audiovisuales-. Hasta aquí, los datos responden al primer y segundo de los objetivos propuestos en esta investigación.

En relación con el tercer objetivo, el componente temático de índole política se impone en el contenido sobre la campaña previa a las elecciones catalanas del 21D, siendo la presencia de infoentretenimiento casi residual. Este aspecto resulta coincidente con la tendencia observada por Berrocal et al. $(2016 ; 2017)$ que apuntan hacia un descenso considerable del politaiment a favor de contenidos institucionales durante periodos electorales.

Respecto al último de los objetivos propuestos, el fenómeno de la personalización política en el caso particular de la contienda electoral examinada se constata en la información producida y consumida en YouTube. El consumidor presta especial atención a los actores protagonistas de las elecciones del 21D (véanse Puigdemont y Arrimadas en esta investigación) y a aspectos que hagan referencia a sus atributos personales. Los candidatos se posicionan por encima de 
las marcas respectivas de sus formaciones y despiertan mayor interés en el consumidor que las propuestas programáticas de los diferentes partidos. En este caso, las evidencias obtenidas resultan coincidentes con los hallazgos de Berrocal et al. (2017) y cuestionan los resultados de Berrocal et al. (2016). Encontramos una posible explicación a esta disparidad en relación al fenómeno de la personalización política en contenidos difundidos en YouTube sobre periodos electorales, en el hecho de que el estudio de Berrocal et al. (2016) se refiere a la participación de partidos políticos españoles en unas elecciones al Parlamento Europeo, una cámara cuyos miembros, al menos en el contexto español, tienen poco eco mediático y con quienes la ciudadanía está menos familiarizada; mientras que el estudio de Berrocal et al. (2017) versa sobre las elecciones generales en España de 2016. En el caso particular de esta investigación, pese a tratarse de unos comicios en un contexto geográfico autonómico, normalmente con menor repercusión mediática y social que unas nacionales, el denominado Procés que precedió la campaña, situó los comicios del 21D en el foco de los medios de comunicación y del debate público nacional, poniendo en primer plano a los líderes de las formaciones que pugnaban por el Parlament.

Esta investigación arroja nuevas evidencias sobre el rol que juega la esfera política en una red social, YouTube, hasta el momento, la menos explorada a nivel académico. Los resultados obtenidos suponen una aportación significativa a la incipiente línea de estudio que aborda la comunicación política en YouTube, pero denotan igualmente la necesidad de que futuras investigaciones contribuyan a reforzarla. Avanzar en esta cuestión permitirá definir los cambios que esta red social está introduciendo tanto en la gestión de la comunicación política como en el consumo de la misma.

\section{Notas}

${ }^{1}$ Para profundizar en datos de audiencia de los debates electorales previos a las elecciones del 21D se puede consultar en: http://vertele.eldiario.es/audiencias-canales/analisis-espana/audiencias-debateselecciones-catalunya-tve-la1-lasexta-tv3_0_1968403151.html [Última consulta: 8 de enero de 2018].

${ }^{2}$ Para conocer más detalles sobre el incremento de audiencia en TV3 se puede consultar en:

https://politica.elpais.com/politica/2017/10/25/actualidad/1508927384_009455.html [Última consulta: 8 de enero de 2018].

${ }^{3}$ Youtube.com es el sitio web (por encima de Facebook.com y Twiteer.com) que acumula mayor número de visitas únicas según el estudio "Audiencia de Internet" (AIMC, 2018).

${ }^{4}$ Por tal entendemos un lenguaje con mensajes directos, cortos y protagonizados por elementos audiovisuales o gráficos llamativos.

${ }^{5}$ Véase apartado 4.1.1

${ }^{6}$ Véase: https://www.youtube.com/watch?v=J29FShGv4oc [Última consulta: 13 de enero de 2018].

${ }^{7}$ Por ello entendemos: aquellos vídeos cuya imagen ha sido grabada en origen por el usuario para 
publicarlo ad hoc en la Red, los que emplean imágenes generadas por el propio usuario tipo stop motion o infografías, o aquellos que, apropiándose de materiales de otros usuarios, realizan modificaciones sobre el audiovisual tipo montajes, rotulaciones o locuciones.

${ }^{8}$ Véase: https://www.youtube.com/watch?v=C4MsFbRcGY4 [Última consulta: 30 enero de 2018].

${ }^{9}$ Véase: https://www.youtube.com/watch?v=a6B9jyzR70w [Última consulta: 30 enero de 2018].

${ }^{10}$ Véanse: https://www.youtube.com/watch?v=1vzaQMfGoRE y https://www.youtube.com/ watch?v=dFNOvRfq-0A [Última consulta: 30 enero de 2018].

${ }^{11}$ Véanse a modo de ejemplo: https://www.youtube.com/watch?v=lfFMuRO4fc8 y https://www.youtube. com/watch?v=DZBIInhaJt8 [Última consulta: 30 enero de 2018].

${ }^{12}$ Véase a modo de ejemplo: https://www.youtube.com/watch?v=qRGIt1zS39o [Última consulta: 30 enero de 2018].

${ }^{13}$ Véase a modo de ejemplo: https://www.youtube.com/watch?v=apWUciX7RRw\&has_verified=1 [Última consulta: 30 enero de 2018].

${ }^{14}$ Véase el apartado 1.3.

\section{Referencias}

Abad, J. M.; Galán, J. y Alameda, D. (20 de diciembre de 2017). Los tuits de los candidatos que más éxito han tenido en campaña. EI PAIS. Recuperado de https://goo.gl/CrVFxx

Abejón, P. y Mayoral, J. (2017). Persuasión a través de Facebook de los candidatos en las elecciones generales de 2016 en España. El profesional de la información, 26 (5), 928-936. https://doi. org/10.3145/epi.2017.sep.14

Abejón, P.; Sastre, A. y Linares, V. (2012). Facebook y Twitter en campañas electorales en España, en Anuario Electrónico de Estudios en Comunicación Social Disertaciones, 5 (1). Artículo 7. Recuperado de https://goo.gl/PsB3sj

Acceso (2017). 21D: La campaña electoral en redes sociales. Recuperado de https://goo.gl/9WfqWz

Almansa, A. y Castillo, A. (2014). Comunicación Institucional en España. Estudio del uso que los diputados españoles hacen de las TIC en sus relaciones con la ciudadanía. Chasqui (126), 22-30.

Alonso, L. y Casero, A. (2018). Political agenda on Twitter during the 2016 Spanish elections: issues, strategies, and users' responses. Communication \& Society 31 (3), 7-23.

Alonso, L., Marcos, S. y Casero, A. (2016). Political leaders in (inter)action. Twitter as a strategic communication tool in electoral campaigns. Trípodos, 39, 71-90.

Aparaschivei, P. A. (2010). The Use of Media in Electoral Campaigns: Analysis on the Use of Blogs, Facebook, Twitter and YouTube in the 2009 Romanian Presidential Campaign. Journal of Communication and Public Relations, 12, 3 (20). 
Aparici, R. y García-Marín, D. (2018). Prosumidores y emirecs: Análisis de dos teorías enfrentadas. Comunicar, 26 (55), 71-79. https://doi.org/10.3916/C55-2018-07

Asociación para la Investigación de Medios de Comunicación (AIMC). (Marzo 2018): Navegantes en la Red. Recuperado de https://bit.ly/2KI8Mhp

Ballesteros, C. A. y Díez Garrido, M. (2018). Tenemos que hablar. El compromiso 2.0 en Facebook durante la cibercampaña española del 20D de 2015. Communication \& Society, 31 (1), 169-193.

Berrocal, S. (2003). Personalización de la Política, en S. Berrocal (Coord.), Comunicación Política en Televisión y Nuevos medios (pp. 55-79). Barcelona: Ariel.

Berrocal, S.; Campos, E. y Redondo, M. (2012a). El “infoentretenimiento" político en Internet: La presencia de cinco líderes europeos en YouTube. RIPS, 11 (4), 107-131. Recuperado de https://goo.gl/ybn5zJ

Berrocal, S.; Campos, E. y Redondo, M. (2012b). Comunicación política en internet: La tendencia al infoentretenimiento político en YouTube. Estudios sobre el Mensaje Periodístico, 18 (2), 653-659. https://doi.org/10.5209/rev_ESMP.2012.v18.n2.41037

Berrocal, S. y Campos, E. (2012c). El infoentretenimiento político en internet. Un análisis de los videos más vistos en YouTube de José Luis Rodríguez Zapatero y Mariano Rajoy, en S. Berrocal y E. Campos (Coord.), La investigación en periodismo político en los nuevos medios (pp. 75-90). Salamanca: Sociedad Española de Periodística.

Berrocal, S.; Campos, E. y Redondo, M. (2014). Prosumidores mediáticos en la comunicación política, el 'politainment' en YouTube. Comunicar, 43, 65-72. https://doi.org/10.3916/C43-2014-06

Berrocal, S.; Gil, A. y Campos, E. (2016). El uso de YouTube en las elecciones al Parlamento Europeo 2014. El caso de España. Comunicación y Hombre, 12, 57-72. Recuperado de https://goo.gl/xgaXQn

Berrocal, S.; Martín, V. y Gil, A. (2017). Líderes políticos en YouTube: información y politainment en las elecciones generales de 2016 (26J) en España. El profesional de la Información, 26 (5), 937-946. https://doi.org/10.3145/epi.2017.sep.15

Borah, P.; Fowler, E. y Ridout, T.N. (2018). Television vs. YouTube: political advertising in the 2012 presidential election, Journal of Information Technology \& Politics, 15 (3), 230-244. https://doi.org/ 10.1080/19331681.2018.1476280

Casero-Ripollés, A. (2010). ¿El despertar del público?: comunicación política, ciudadanía y web 2.0, en M. Martín y D. Rothberg (Eds.), Medios de comuniçao e ciudadanía (pp. 107-122). Sao Paulo: Cultura académica.

Casero-Ripollés, A. (2018). Research on political information and social media: Key points and challenges for the future. El profesional de la información, 27 (5), 964-974. https://doi.org/10.3145/epi.2018. sep.01

Castells, M. (2012). Networks of outrage and hope: social movements in the Internet age, Cambridge: Polity Press. 
Catalina-García, B., López de Ayala, M.C. y Fernández-Fernández, J.G. (2015). Twitter como plataforma de los alcaldes para la comunicación pública. Estudios sobre el Mensaje Periodístico, 21 (2), 757772. http://dx.doi.org/10.5209/rev_ESMP.2015.v21.n2.50884

Chadwick, A. (2017). The hybrid media system: Politics and power. New York: Oxford University Press.

Cheong, P. y Lundry, C. (2012). Prosumption, Transmediation, and Resistance: Terrorism and Manhunting in Southeast Asia. American Behavioral Scientist, 56 (4), 488-510. https://doi. org/10.1177/0002764211429365

Church, S.H. (2010). YouTube Politics: YouChoose and Leadership Rhetoric During the 2008 Election, Journal of Information Technology \& Politics, 7 (2-3), 124-142. https://doi.org/10.1080/19331681003748933

Criado, J. I., Martínez-Fuentes, G. y Silván, A. (2013). Twitter en campaña: la elecciones municipales españolas de 2011. RIPS, 12 (1), 93-113.

Coromina, O.; Prado, E. y Padilla, A. (2018). The grammatization of emotions on Facebook in the elections to the Parliament of Catalonia 2017. El profesional de la información, 27 (5), 1004-1011. https:// doi.org//10.3145/epi.2018.sep.05

Dader, J. L. (1990). La personalización de la política, en A. Muñoz; C. Monzón; J. I. Rospir y J. L. Dader (Eds), Opinión pública y comunicación política. Madrid: Eudema.

Dader, J.L. (2009). Ciberpolítica en los websites de partidos políticos. La experiencia de las elecciones de 2008 en España ante las tendencias transnacionales. Revista de Sociologia e Política, 17 (34), 45-62.

De Moragas, M. (2011). Interpretar la comunicación. Estudios sobre medios en América y Europa. Barcelona: Gedisa.

Deltell, L. (2012). Estudio del uso de Twitter, Facebook y YouTube en la campaña electoral de 2011 en España. El insólito caso de eQuo, en VV.AA, Crisis y Cambios en las sociedades contemporáneas: retos teóricos y prácticos. Madrid: UCM.

Deuze, M. (2007). Convergence culture in the creative industries, en International Journal of Cultural Studies, 10 (2), 243-63. Recuperado de https://goo.gl/Zb8vni

Espino, G. (2014). La política en Internet, ¿de la mediatización a la convergencia digital?, en Convergencia. Revista de Ciencias Sociales, 65, 39-63. Recuperado de https://goo.gl/YxHVRJ

Feenstra, R. A. y Casero-Ripollés, A. (2012). Nuevas formas de producción de noticias en el entorno digital y cambios en el periodismo: el caso del 15-M, en Comunicación y Hombre, 8, 128-140. Recuperado de https://goo.gl/yPyUYD

Fenol, V. y Cano, L. (2017). Participación ciudadana en los perfiles de Facebook de los partidos españoles. Análisis de comentarios en la campaña electoral de 2015. Communication \& Society, 30 (4), 131148. 
Gil, J. (2015). Cuando el 15M envejeció a los medios de comunicación: emergencia de prosumidores y desarrollo de nuevos modelos mediáticos, en Aposta. Revista de Ciencias Sociales, 66, 9-37. Recuperado de https://goo.gl/9TeSAn

Graham, T., Broersma, M., Hazelhoff, K. y van 't Haar, G. (2013). Between broadcasting political messages and interacting with voters: The use of Twitter during the 2010 UK general election campaign. Information, Communication \& Society, 16 (5), 692-716. doi:10.1080/1369118X.2013.785581

Intelligence Compass (2011). Informe sobre política y Redes Sociales. Recuperado de https://goo.gl/ A2sngF

Jenkins, H. (2006). Convergence Culture. Where old and new media collide. Nueva York: New York Press.

Jivkova, D., Requeijo, P. y Padilla, G. (2017). Usos y tendencias de Twitter en la campaña a elecciones generales españolas del 20D de 2015: hashtags que fueron trending topic. El profesional de la Información, 26 (5), 824-837. https://doi.org/10.3145/epi.2017.sep.05

Lilleker, Darren, G. y Nigel, J. (2009). Politicians and Web 2.0: the current bandwagon or changing the mindset? Paper presented at the Politics: Web 2.0 International Conference, April 17-18, 2008. London: Royal Holloway, University of London.

López-Abellán, M. (2012). Twitter como instrumento de comunicación política en campaña: Elecciones generales 2011. Cuadernos de gestión de información, (2), 69-84. Recuperado de https://bit. ly/2OpvSMh

López-Meri, A. (2016). Twitter-retórica para captar votos en campaña electoral. El caso de las elecciones de Cataluña de 2015. Comunicación y Hombre, n 12, 97-118.

López-Meri, A., Marcos, S. y Casero, A. (2017). What do politicians do on Twitter? Functions and communication strategies in the Spanish electoral campaign of 2016. El profesional de la Información, 26 (5), 795-804. https://doi.org/10.3145/epi.2017.sep.02

Mascheroni, G. y Matoni, A. (2012). Electoral campaigning 2.0 -The case of 2010 Italian Regional Elections. Journal of Information Technology \& Politics, 10 (2), 223-240. https://doi.org/10.1080/19331681.2 012.758073

McNair, B. (2006). Cultural Chaos. Journalism, news and power in a globalised world. Londres: Routledge.

Miquel, S., Alonso, L. y Marcos, S. (2017). Buscando la interacción. Partidos y candidatos en Twitter durante las elecciones generales de 2015. Prisma Social, nº18, 34-54.

Muñiz, C., Dader, J.L., Téllez, N.M. y Salazar, A. (2016). ¿Están los políticos políticamente comprometidos? Análisis del compromiso político 2.0 desarrollado por los candidatos a través de Facebook. Cuadernos.info, (39), 135-150. https://doi.org/10.7764/cdi.39.970

Pérez-Curiel, C. y García-Gordillo, M. (2018). Política de influencia y tendencia fake en Twitter. Efectos postelectorales (21D) en el marco del Procés en Cataluña. El profesional de la información, 27 (5), 1030-1040. https://doi.org/10.3145/epi.2018.sep.07 
Polo, D. y Cárdenas, M. L. (2014). Infoxicación y Tweets. Análisis del Caso Bárcenas a través del Twitter de Mariano Rajoy, en Ámbitos, 26. Recuperado de https://goo.gl/gmGHGU

Quevedo, R; Portalés, M. y Berrocal, S. (2016). El uso de la imagen en Twitter durante la campaña electoral municipal de 2015 en España. Revista Latina de Comunicación Social, 71, 85-107. https://doi. org/10.4185/RLCS-2016-1085

Rodríguez-Breijo, V.; Gallardo, J. y Sierra, J. (2018). Información política en los vídeos que son tendencia en YouTube España. El profesional de la información, 27 (5), 1041-1049. https://doi.org/10.3145/ epi.2018.sep.08

Rodríguez-Fidalgo, M. I., Ruiz Y., Paíno, A., Jiménez Iglesias, L. (2017). El uso de Facebook como herramienta narrativa para articular el nuevo diálogo político. Estudio de caso de la II Asamblea Ciudadana de Podemos, "Vistalegre II". Revista Latina de Comunicación Social, (72), 849-860.

Sampedro, V. y Resina, J. (2010). Opinión pública y democracia deliberativa en la Sociedad Red, en Ayer, 80 (4), 139-62. Recuperado de https://goo.gl/5kUop5

Schweitzer, E. J. (2012). The Mediatization of E-Campaigning: Evidence From German Party Websites in State, National, and European Parliamentary Elections 2002-2009. Journal of computer-mediated communication, 7 (3), 283-302. https://doi.org/10.1111/j.1083-6101.2012.01577.x

Sohal, S. y Kaur, H. (2018). A Content Analysis of YouTube Political Advertisements: Evidence from Indian Parliamentary Elections. Journal of Creative Communications, 13 (2), 133-156. https://doi. org/10.1177/0973258618761408

Toffler, A. (1980). The Third Wave. Nueva York, USA: Bantam Books.

Túñez, M. y Sixto, J. (2011). Redes sociales, política y Compromiso 2.0: 'La comunicación de los diputados españoles en Facebook'. Revista Latina de Comunicación Social, 66, 210-246. https://doi. org/10.4185/RLCS-66-2011-930-210-246

Varona, D.; Sánchez, M.; Arrocha, R. (2017). Consumo de información política en dispositivos móviles en España: caracterización del usuario tipo y su interacción con las noticias. El profesional de la Información, 26 (4), 641-648. https://doi.org/10.3145/epi.2017.jul.08

Vázquez-Sande, P. (2016). Storytelling personal en política a través de Youtube. Comunicación y Hombre, 12, 41-55.

Vesnic-Alujevic, L. y Van Bauwel, S. (2014). YouTube: A Political Advertising Tool? A Case Study of the Use of YouTube in the Campaign for the European Parliament Elections. Journal of Political Marketing, 13 (3), 195-212. https://doi.org/10.1080/15377857.2014.929886 